\title{
Prolonged Fever And Acute Abdomen as a Rare Manifestation of Tubo-Ovarian Tuberculosis : A Case Report
}

\author{
Angga Maulidha ${ }^{2}$, Bramantono ${ }^{1}$, Usman Hadi ${ }^{1}$ \\ Division of Tropical and Infectious Disease ${ }^{1}$ \\ Departement of Internal Medicine ${ }^{2}$ \\ Faculty of Medicine Universitas Airlangga, \\ Dr.Soetomo General Hospital, Surabaya, Indonesia
}

\begin{abstract}
Introduction: Female genital tuberculosis is a rare form of extrapulmonary TB. It may lead to delayed diagnosis because of nonspecific features such as fever, abdominal distension, ascites, and weight loss. Objective: To reported a case of prolonged fever and acute abdomen due to turbo-ovarian tuberculosis. Case Presentation: A female 22 years old came to ER with a major complaint of right lower quadrant abdominal pain for 2 days. The patient has medical checkup history at the internal medicine clinic with a complaint of prolonged fever for 2 months, decrease body weight by $7 \mathrm{~kg}$ in 3 months, and abdominal tenderness. She has a negative result for HIV, hepatitis, salmonella and TORCH work up. Physical examination in the ER found slightly distended abdomen, McBurney sign (+), and subfebrile. Laboratory result showed mild anemia $(9,5)$, negative pregnancy test and elevated CRP and LDH. Abdominal Doppler USG result free fluid intra-abdomen and cystic mass in the right pelvis impressive an ovarian mass. CT scan with triple contrast confirmed there's bilateral cystic mass with diameter $\pm 11 \mathrm{~cm}$ (right) and $\pm 7 \mathrm{~cm}$ (left) with feeding artery from artery ovarica refer to malignant ovarian cyst. Discussion: The patient took laparotomy, and during operation found attachment of right tubo-ovarian with adnexa and active bleeding. The pathological expertise described necrotic tissue with granulomatous formation due to tuberculosis. Anti TB drugs were given for 9 months, and the patient showed clinical improvement with reduction of ovarian size. Conclusion: Tubo-ovarian tuberculosis should be considered in the differential diagnosis of lower quadrant pain with decrease body weight in a young female who live in tuberculosis-endemic regions.
\end{abstract}

Published by IJRP.ORG. Selection and/or peer-review under responsibility of International Journal of Research Publications (IJRP.ORG)

Keywords: prolonged fever, acute abdomen, ovarian, tuberculosis

\section{Introduction}

Fever is the most common presenting symptom that bring the patients came to clinicians. Based on its duration, fever can be classified into acute $(<7$ days in duration), subacute $(<2$ weeks in duration), and 
chronic ( $>2$ weeks duration). Chronic fever is typically form of bacterial infections such as tuberculosis, viral infections like HIV, cancers, and connective tissue diseases (Ogoina, 2011). The chronic fever could develop into prolonged fever and terminologically called Fever of unknown origin (FUO) if the disease condition remains for at least three weeks, with no diagnosis made despite one week of inpatient investigation (Unger, Karanikas, Kerschbaumer, Winkler, \& Aletaha, 2016).

Prolonged fever followed by abdominal pain should be suspected as abdominal tuberculosis at any age group. Even though abdominal pain become the main symptom of abdominal tuberculosis, acute abdomen as a manifestation of genital tuberculosis is rare. Genital tuberculosis affects about $12 \%$ of women with Pulmonary TB and $20 \%$ of women with extrapulmonary TB (Reports, Akbulut, Arikanoglu, \& Basbug, 2011). Female genital TB is an important cause of significant morbidity especially infertility. The most affected organ is the fallopian tubes (90-100\% cases). The clinical presentation of female genital TB depends on the site of the genital organs involved. Mostly, asymptomatic or few symptoms which infertility is the most frequent. In this case, the manifestation of genital TB is the acute abdomen, which is a rare manifestation. The worldwide incidence of acute abdomen suspected appendicitis who present normal appendices ranges from $7.7 \%$ to $54 \%$ (Kutasy B, et al, 2010). A variety of gynecological diseases can imitate acute abdominal symptoms, including tubal ectopic pregnancy, torsion of the ovarian, tubo-ovarian masses, or infected cysts, pelvic inflammatory disease (PID), and granulomatous causes such as tuberculosis (TB). The study result from Egypt explain mostly abdominal tuberculosis present at young productive age, $73 \%$ of the patients with acute abdominal tuberculosis were below 40 years, and $45.5 \%$ of the patients were between 20 40 years, with a female to male tratioof 1:1,25.

Diagnostic of female genital tuberculosis is often delayed because of the nonspecific feature. Various investigation methods had been disclosed as the gold standard in previous studies. The diagnosis is made by finding the acid-fast bacilli on the biopsy specimen. The polymerase chain reaction could be false-positive and not common to make the diagnosis. While, laparoscopy or hysteroscopy can diagnose genital tuberculosis by heterogenous finding (Sharma, 2015). Thus in an endemic area, the differential diagnosis of acute abdomen in a young female with prolonged fever and decreased bodyweight should be considered as abdominal tuberculosis (Science, 2018). Here we report a case of prolonged fever and acute abdomen in a young female patient as a manifestation of tubo-ovarian tuberculosis.

\section{Case Description}

A female 22 years old came to ER with a major complaint of right lower quadrant abdominal pain 2 days before and getting worse. The pain is felt continuous. She told that her stomach appear enlarged since 2 weeks before, painless, sometimes felt uncomfortable, She was thought that it just constipation because she hadn't got defecation for about 1 week also complained of fever since 2 months ago, subfebrile, getting better with antipyretic. She confirmed a decrease bodyweight for about $7 \mathrm{kgs}$ in 2 months. She is unmarried yet, her history of the menstrual problem is denied. There is no problem with urinary habit. History of past examination: The patient had examined this prolonged fever complaint to the clinic and obtained normal CBC and urinalysis, negative Salmonella, negative ANA test, negative hepatitis B and C, negative HIV rapid test. Past chest X-ray examination was normal, then the patient was given antipyretic drugs, antibiotics, and vitamins. History of chronic cough for 3 weeks in 7 month ago, and the symptom disappear after taken 
medication from the doctor. Psychosocial life : The patient is a college student, living in an Islamic boarding house.

The general condition was weak, GCS 456, underweight (BMI 18,7 kg/m² ) BP 130/90, HR 108x/ min regularly, $\mathrm{RR} 20 x / \mathrm{min}$, temperature $38,3^{\circ} \mathrm{C}, \mathrm{O}^{2}$ saturation $98 \%$ free air , VAS $7 / 10$. There was anemia, There was no abnormality detected in lung and heart examination. From the abdominal examination, we found slight distended, dull pain in the right lower quadrant, bowel sound increased, tympani, Mc Burney pain positive, Patrick and Contra Patrick's sign positive, obturator sign positive. Laboratory result showed decreased $\mathrm{Hb}$ (9,5 d/dL), increased LDH 299, CRP $20.06 \mathrm{mg} / \mathrm{L}$, non specific increased tumor marker Ca-125 $(36,1)$. The other laboratory finding was normal. We also didn't see any abnormality on the left lateral decubitus Abdominal x-ray. The abdominal sonography found some intra-abdominal free fluid and cystic mass in the right pelvis, impress an ovarian mass.

The patient consulted to Obstetric/Gynecologic (OG) and digestive surgery because of complaining acute abdomen with those supporting results. The Digestive team assessed colic abdomen suspecting periappendicular infiltrat (PAI) differential diagnosing with ovarian mass. They suggested triple contrast CT scan abdomen examination. From OG departement they diagnosed acute abdomen et cause ovarian cyst strangulation dd PAI and suspect peritonitis. They suggested CT abdomen with triple contrast, CEA, Ca-125, Pregnancy test, and AFP. From that data, the patient diagnosed with Acute abdomen et causa ovarial mass strangulata with differential diagnosis Periappendicular infiltrat and suspected early peritonitis. After that she got hospitalized and prepared to do emergency Abdominal CT triple contrast to confirm the diagnosis. The abdominal CT scan with triple contrast found bilateral multiloculated cystic lesions with septa, size $7 \times 5.2 \times$ $8.6 \mathrm{~cm}$ with feeding from artery ovarica sinistra. And the left one size $11 \times 4.2 \times 5.9 \mathrm{~cm}$ that received feeding from a.ovarica dextra, septal's thickness $0.6 \mathrm{~cm}$, which contrast enhancement to the wall, fat stranding $(+)$, attached to the bladder, uterus and adjacent bowel suspected a malignant ovarial mass. Subcentimeter lymphnodes in paraaorta, aortocava, mesenterial lymphadenopathy left and right iliaca and with no sign of appedicitis

Pre-operation laboratory found anemia with $\mathrm{Hb} 8.2$, suspecting internal bleeding from its malignant ovarial mass bilateral. Symptomatic treatment also had given to patient for her abdominal pain. Laparotomy procedure was performed by gynecologyst and digestive surgeon. The surgeon reported attachment of right tubo-ovarian with bladder and adnexa, they also found active bleeding from the right tuba fallopian adhessive site, there was no abnormality found from appendix site. The right ovarian mass is taken by debulking procedure and ligating the source of bleeding. An ovarian mass with diametre size $12 \mathrm{~cm}$ brought to the pathological expertise. After $4^{\text {th }}$ day of hospitalization, the patient told that the abdominal pain was reduced, she is in a good condition. TD $110 / 70 \mathrm{HR} 76 \mathrm{x} / \mathrm{min} \mathrm{RR} 18 \mathrm{x} / \mathrm{min}$, temperature $36^{\circ} \mathrm{C}$. laboratory finding $\mathrm{Hb}$ 10,4 g/dL, WBC 8.320 , Plt 403.000. Patient got discharged and got medication tramadol tablet 1 tablet if needed. The patient was scheduled to get wound care and took the pathological result at outpatient department.

On the first outpatient examination, the patient brought pathological result that described with ovarian tissue composed by epithelioid-shaped cells of histiocytes, partially clustered around the necrosis area become granulomatous change. Distribution of multi nucleated giant cell datia langhans and large areas of necrosis. No signs of malignancy. In conclusion as a granulomatic inflammation from tuberculosis in the fallopian tube and ovarium. According to the pathological result, the patient diagnosed with Tubo-ovarian tuberculosis. She was referred to the tuberculosis center to get anti TB Drugs (ATD). She got first category ATD for 9 months (3 tabs 4FDC/3 tabs 2FDC) after gene Xpert examination showed no resistence on rifampicin, and then she was planned to take an abdominal CT evaluation after 2 months of intensive phase of ATD. 
After 2 months took intensive phase of ATD, the patient showed a good clinical respons. Her body weight increased from $46 \mathrm{~kg}$ to $52 \mathrm{~kg}$ in two months, there is no complaint about menstrual cycle nor abdominal pain. The CT abdominal evaluation's showed reduce of left ovarian mass. In the last month of ATD treatment, the patient condition was good with a significant increasing body weight total $15 \mathrm{~kg}$ after ATD treatment. Her BMI has back to the ideal $\left(24,7 \mathrm{~kg} / \mathrm{m}^{2}\right)$.
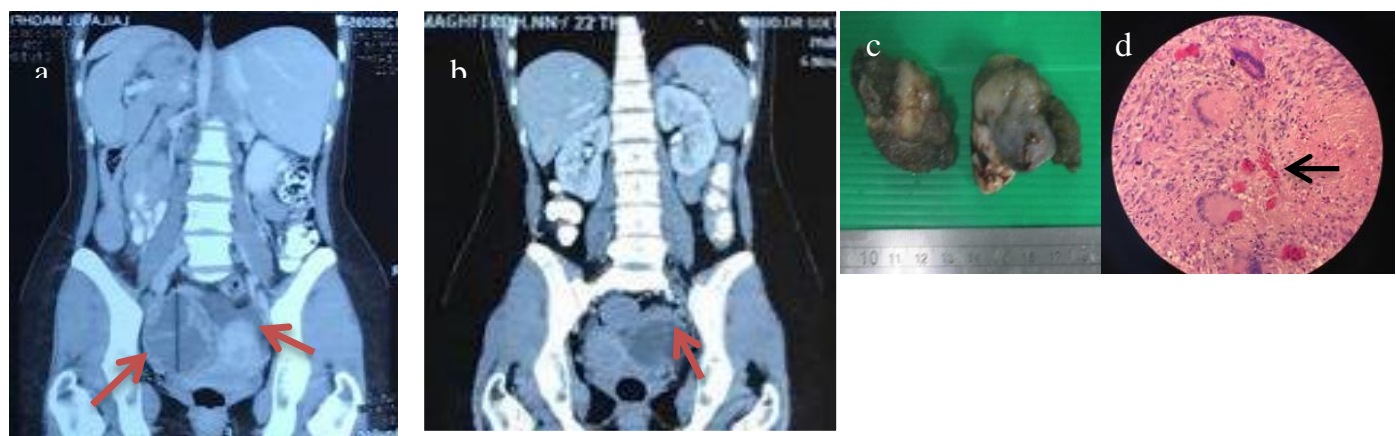

Figure 1. a) Abdominal CT showed bilateral cystic mass of the ovarian size $11 \times 4,2 \times 5,9 \mathrm{~cm}$ (right mass) and $7 \times 5,2 \times 8,6$ (left mass), with feeding artery from a.ovarica refer to malignant ovarial cyst. b) Abdominal CT evaluation after 2 months taking Anti-TB drugs, left ovarian's size decreased $6 \times 2 \times 7 \mathrm{~cm}$. c)Macroscopic view of the tissue taken from adhesive tissue around right adnexa. d)Pathological result : red stain baccili, necrotic caseation, granulomatous formation, langhans giant cell $(+)$ typical tuberculosis form.

\section{Discussion}

According to The International Union of Physiological Sciences Commission for Thermal Physiology in 2001, fever defined as state of elevated core temperature, which is part of the defensive responses of host to the invasion of live (micro-organisms) or pathogenic or alien by the host. Based on its duration, fevers can be classified into acute, sub-acute and chronic. Acute fevers occurs less than 7 days, the etiology commonly from parasitic infections such as malaria and viral-related upper respiratory tract infection. Sub-acute fevers usually occurs not more than 2 weeks, it could be seen in typhoid fever and intra abdominal abscess. Persistent or chronic fevers occurs more than 2 weeks duration, they are typical of chronic infections such as HIV, tuberculosis, malignancy and rheumatologic diseases (Ogoina, 2011).

Based on it's types, there are three major fever including intermittent fever, remittent fever and continuous fever. Intermittent fever describe as fever present only for several hours during the day. This pattern can be found in malaria, tuberculosis, schistosomiasis, pyogenic infections, leptospira, lymphomas, borrelia, and Kalaazar disease. Remittent fever is defined as fever with daily fluctuations outreach $2{ }^{\circ} \mathrm{C}$ but never touches normal. Remittent fevers usually correlated with infectious diseases such as infective 
endocarditis, brucellosis and rickettsiosis. Fever followed with night sweats was typically related with TB, and atypically found in liver or lung abscess, brucellosis, and sub-acute infective endocarditis. Continuous or sustained fever is determine as fever that does not fluctuate more than about $1{ }^{\circ} \mathrm{C}$ during the day, but never touches normal temperature. Continuous fevers are typical form of typhoid, lobar and gram-negative pneumonia, urinary tract infection and acute bacterial meningitis (Ogoina, 2011).

In the investigation of fever of unknown origin (FUO), the presence of low grade intermittent fever and night sweats without localising sign is a key point to consider extrapulmonary tuberculosis, or to initiate empirical anti-tuberculosis therapy in tuberculosis endemic regions (Ongoina, 2011). In this case we found that the patient had a fever lasted for 2 months. Based on its onset, this fever was classified as chronic fever. From the discussion above, there is a differential diagnosis of chronic fever including tuberculosis, HIV infection, malignancy and autoimmune disease. Fever in this case could be classified as intermittent fever because it often appears in the evening or night. Even though there was no temperature measurements were taken, the anamnesis data stated that fever was low grade or sub-febrile. Fever usually get better when she wake up in the morning without medication (Ogoina, 2011).

Based on this basic diagnostic approach, there is some controversies about the next diagnostic panel. Most scientists will use a broad diagnostic approach including blood tests, urinalysis, culture, stool tests, skin tests, x-rays, and abdominal ultrasound. If the diagnosis is still not defined, some suggests hepatitis serology, igD serum, liver biopsy, fungal culture, echocardiography, abdominal and thoracic CT scans, colon x-ray and temporal biopsy for those over 55 years of age. Likewise, procalcitonin testing is also often used to distinguish between infectious and non-infectious causes (Ogoina, 2011).

Empiric antibiotics are not recommended in cases of prolonged fever because they can disguise and delay the diagnosis. However, there are several suggestions for initiating empirical therapy such as the use of antibiotics in suspected negative culture-infective endocarditis, suspected active tuberculosis, and glucocorticoids for suspected temporal arteritis with the risk of vision loss. Antipyretic therapy can be a special form of empirical therapy because it does not need require the etiology of fever. Antipyretics can be given to patients who are unable to tolerate the fever peaks. For diagnostic purposes, antipyretics have been reported to differentiate fever from malignancy and infection although their sensitivity and specificity are low. In fever due to malignancy, may respond up to normal temperature, while for fever due to infection, antipyretics may not show a response. Acute abdominal pain has a very wide diagnostic spectrum ranging from minor causes to surgical emergencies. The location and characteristic of the pain can be used as an examination to establish the diagnosis. For example, right lower quadrant pain is specific for acute appendicitis. In addition, it is necessary to consider special populations such as women who are at risk of 
developing genitourinary disease (Forest \& Carolina, 2008).

Our patient complained of a right lower quadrant abdominal pain since 2 days before admission and getting worse. The pain is continuously felt and decreased with the bend position. The patient also complained of an enlarged abdomen and defecated bowel movements. From the physical examination we found a positive McBurney pain, Patrick and Contra Patrick's positive sign, this condition includes acute abdominal symptoms. Of the problems found in patients, the diagnosis leads to acute appendicitis, but acute abdomen in women must also consider the causes of genital organs such as ectopic pregnancy or twisted cysts. So that the next examination is performed pregnancy tests and abdominal ultrasound to rule out the differential diagnosis. Urine pregnancy test results were negative but the ultrasound results ovarian cysts and free fluid. So that the diagnosis leads to a twisted right ovarian cyst with perforation suspected. The surgery reported attachment of right tubo-ovarian with bladder and adnexa, they also found active bleeding from the right tuba fallopian adhessive site, there was no abnormality found from appendix site. The right ovarian mass is taken by debulking procedure and ligating the source of bleeding. An ovarian mass with diametre size $12 \mathrm{~cm}$ with pathological result that described granulomatic inflammation was seen due tuberculosis in the fallopian tube and ovary.

The most common cause of acute abdomen require emergency surgery is acute appendicitis. There are many differential diagnoses of acute abdomen in the lower quadrant. In women, gynecological diseases such as ectopic pregnancy, tubo-ovarial abscess, pelvic inflammatory disease (PID), and strangulation ovarian cysts should be considered ad differential diagnosis. Among these causes, PID associated with tubo-ovarian abscess due to nisseria gonorrhea, chlamydia, and tuberculous granuloma can be dangerous. Tuberculosis is still the biggest problem in the world. Most cases discovered in Southeast Asia region. In 2016, the report of tuberculosis cases in Indonesia reached 358,608 (91\% pulmonary TB, 8.3\% extra pulmonary TB). Genital TB is found in $12 \%$ of women with pulmonary TB and $20 \%$ in extra pulmonary TB (Reports et al., 2011).

Genital TB in women is generally caused by hematologic or lymphatic spread from pulmonary TB. However, primary genital TB can also occur in women whose partners have active genitourinary TB. The location of primary genital TB in women are usually the cervix, vulva or vagina. Primary transmission can also occur as a result of direct spread from infected abdominal organs, for example in intestinal TB which spreads to the genital organs. Genital tuberculosis in women often occurs at the age of 20-40 years. Prompt diagnosis and prompt treatment can prevent infertility (Sharma, 2015). Genital TB in women lead to important morbidity due to infertility. The most frequent anatomical location involved is fallopian tubes (90$100 \%$ ) with clinical manifestation congestion, hydrosalphynx, pyosalphynx and tubo-ovarian masses. The endometrium is involved in $50-80 \%$ with caseosa, ulcerations and adhesions (Asherman's syndrome). The 
ovaries are involved in 20-30\% cases with manifestations similar to ovarian cancer because it can be followed by increasing of Ca-125 levels. the Cervix involved about 5-15\% of cases. Mostly, symptoms reported are abdominal pain, menstrual disorders (oligomenorrhea, hypomenorrhea, amenorrhea, menorrhagia, dysmenorrhea, metrorrhagia), pelvic pain, and abnormal vaginal discharge. In post-menopausal women, genital TB can be present clinical symptoms similar with endometrial malignancy, such as postmenopausal bleeding or persistent vaginal discharge (Sharma, 2015).

Most of the patients had pelvic masses, ascites and elevated Ca-125 levels. Ca-125 is a non-specific ovarian cancer marker that can be increased in conditions like PID, genital TB, endometriosis, Meige's syndrome, peritonitis, ovarian hyperstimulation, hepatitis and pancreatitis. Previous studies reported that genital TB rarely increases very high Ca-125 levels (> $1000 \mathrm{U} / \mathrm{ml}$ ). Normalization of Ca-125 levels related with a good response to anti-TB therapy (Yessae F \& Farzaenah F, 2009). Radiologic examination (CT scan) of female genital tuberculosis cannot explained the characteristic features, and often mistaken with ovarian malignancy or PID. Kumar et al explained in his study, about 20 from 32 abdominal CT scan examination were misinterpreted as ovarian malignancy, chocolate cyst or PID. The diagnostic procedures that enforced these 20 patients were derived from histopathological preparations from laparotomy or laparoscopy (Kumar et al., 2017). Magnetic resonance imaging (MRI), positron emission tomography (PET) and Ultrasonography (USG) have all been used to convey genital TB in women, but the features are not specific for TB. The main diagnostic modalities are histopathologic from laparoscopic speciemen, or detect adenosine deaminase (ADA) levels in ascitic fluid if available. In addition, positron emission tomography with glucose 18 F-fluorodeoxy (FDG-PET) has been used successfully helped diagnosed peritoneal tuberculosis and tuberculous mass (Kumar et al., 2017). Integrated with clinical history and laboratory tests, pelvic tuberculosis should be considered in young females with poor socioeconomics having slightly elevated Ca-125 and CT findings of adnexal mixed (solid and cystic) mass with multilocular caseous necrotic enhancement, high density ascites, thickened and enhanced peritoneum. Chest CT scan is also helpful to aid in the diagnosis. Preoperative diagnosis of pelvic TB is of paramount importance to avoid misdiagnosis and surgical explorations.

Generally, the management of extra pulmonary TB is divided into medical therapy and surgical therapy. Medical therapy with first-line anti-TB drugs (ATD) is the main therapy. ATD therapy is given using the same regimen as for pulmonary TB therapy, but with a longer treatment period of 9 months. In the initial phase rifampin is given, INH, ethambutol and pyrazinamide for 2 months, then in the advanced phase with rifampicin and INH given for. Within the period of treatment our patient showed improvement condition and quality of life. 


\section{Conclussion}

A case about prolonged fever and acute abdomen as rare manifestation of genital tuberculosis (GTB) in young female has been reported. The delay in the diagnosis of genital tuberculosis resulted in prolonged fever in this patient. The presence of acute abdomen is also great mimicker of other female abdominal conditions such as acute appendicitis, torsion ovarian cyst, or ruptured ectopic pregnancy. The diagnosis of GTB remains challenging, the gold standard for GTB is a specific culture, acid bacillus stain, and PCR TB from intraoperative specimens. The medication of this patient is referred to the Extrapulmonary TB treatment, by giving standard anti-TB (isoniazid, rifampicin, pyrazinamide, and ethambutol) for 2 months, followed by isoniazid and rifampicin alone for another 9 or 12 months. From this case we may take a note that tuboovarian tuberculosis should be considered in the suspected diagnosis of lower quadrant pain with decrease of body weight and in young female who live in tuberculosis-endemic regions.

\section{Acknowledgements}

Author sincerely would like to gratitude to all lectures, family, and the patient for their endless support and prayers.

\section{References}

Kumar, S., Shi, X., Du, S., Li, X., Hua, C., \& Shah, S. (2017). ScienceDirect CT findings and analysis for misdiagnosis of female pelvic tuberculosis. Radiology of Infectious Diseases, 4(1), 19-25. https://doi.org/10.1016/j.jrid.2016.04.001

Ogoina, D. (2011). Fever, fever patterns and diseases called ' fever' — A review. Journal of Infection and Public Health, 4(3), 108-124. https://doi.org/10.1016/j.jiph.2011.05.002

Reports, C., Akbulut, S., Arikanoglu, Z., \& Basbug, M. (2011). Tubercular tubo-ovarian cystic mass mimicking acute appendicitis : a case report. Journal of Medical Case Reports, 5(1), 363. https://doi.org/10.1186/1752-1947-5-363

Sharma, J. B. (2015). Current Diagnosis and Management of Female Genital Tuberculosis. The Journal of Obstetrics and Gynecology of India, 65(6), 362-371. https://doi.org/10.1007/s13224-015-0780-z

Unger, M., Karanikas, G., Kerschbaumer, A., Winkler, S., \& Aletaha, D. (2016). Fever of unknown origin ( FUO ) revised Case report, 796-801. https://doi.org/10.1007/s00508-016-1083-9

Yassaee F, Farzaneh F. 2009 Familial tuberculosis mimicking advanced ovarian cancer. Infect Dis Obstet Gynecol:736018. 
